Supporting Information for:

\title{
Geometry-Induced Asymmetric Vanadium Ion Permeation of PVDF Membranes and Its Effect on the Performance of Vanadium Redox Flow Batteries
}

Seonghyun Yoon,,$\pitchfork$ Eunju Lee, ${ }^{\dagger}, \star$ Sang Jun Yoon, ${ }^{\dagger}$ Duk Man Yu, ${ }^{\dagger}$ Young Jin Kim, ${ }^{\ddagger}$ Young Taik Hong, ${ }^{*} \uparrow$ Soonyong So ${ }^{*} \uparrow$

${ }^{\dagger}$ Energy Materials Research Center, Korea Research Institute of Chemical Technology (KRICT), Daejeon 34114, South Korea

Department of Polymer Science and Engineering, Chungnam National University, Daejeon 34134, South Korea

\section{Corresponding Authors}

Soonyong So

*Email: syso@krict.re.kr (S.S).

Young Taik Hong

*Email: ythong@krict.re.kr (Y.T.H). 


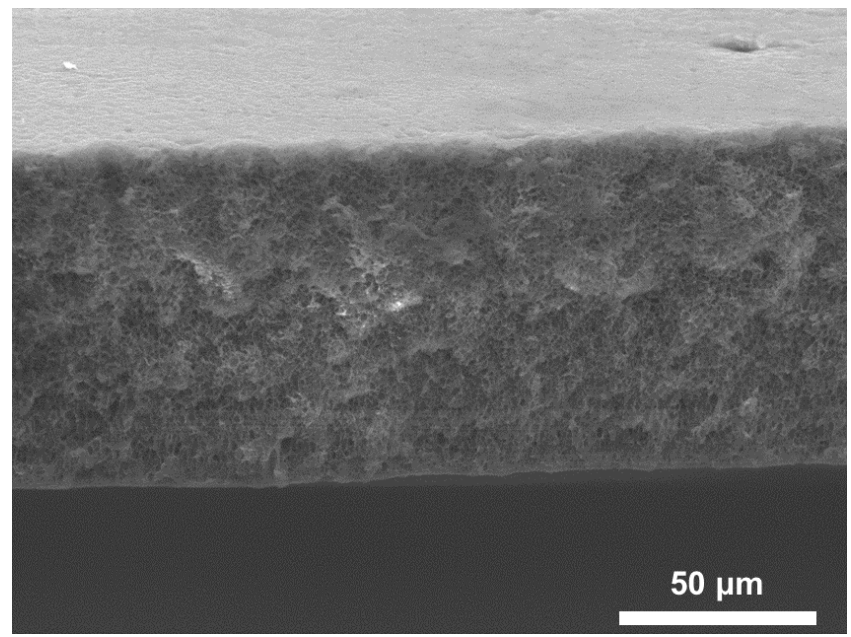

Figure S1. Cross-sectional SEM images of PVDF membranes at $T_{\mathrm{c}}=70^{\circ} \mathrm{C}$. (Scale bars: $50 \mu \mathrm{m}$ )

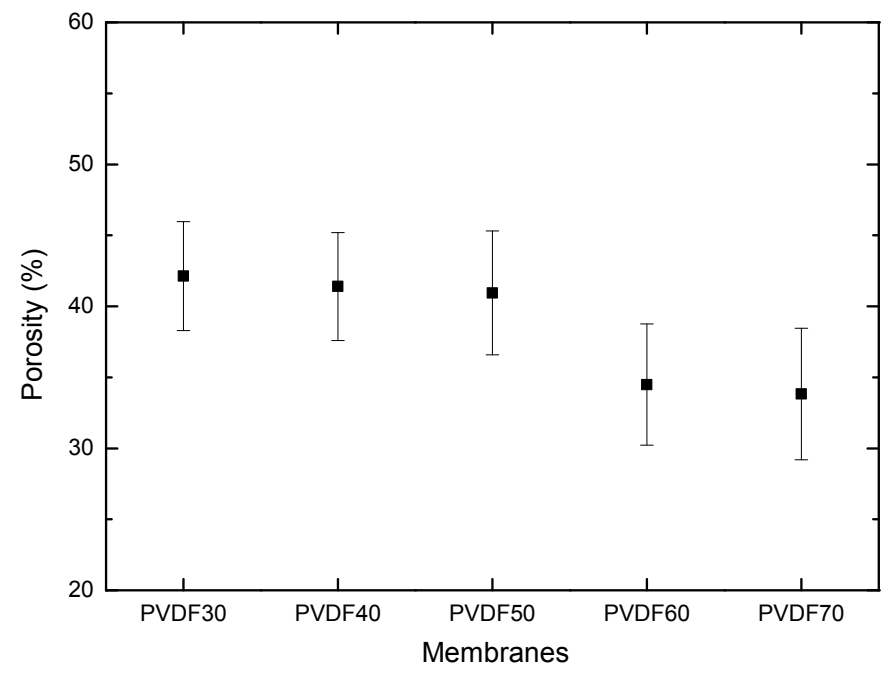

Figure S2. Porosity of the porous PVDF membranes. 
Table S1. Mechanical property of PVDF membranes.

\begin{tabular}{cccc}
\hline Membrane & Modulus (MPa) & Tensile strength (MPa) & Elongation at break (\%) \\
\hline PVDF30 & $820.4 \pm 120.7$ & $12.9 \pm 0.6$ & $716.1 \pm 75.4$ \\
PVDF40 & $1096.1 \pm 86.5$ & $15.9 \pm 1.6$ & $1096.1 \pm 86.5$ \\
PVDF50 & $1140.2 \pm 73.8$ & $18.9 \pm 1.7$ & $874.9 \pm 77.7$ \\
PVDF60 & $1318.8 \pm 101.0$ & $15.8 \pm 3.6$ & $692.8 \pm 190.4$ \\
PVDF70 & $1419.9 \pm 59.0$ & $16.5 \pm 1.1$ & $689.6 \pm 24.1$ \\
\hline
\end{tabular}

Table S2. Vanadium ion permeability and areal resistance of the membranes.

\begin{tabular}{|c|c|c|}
\hline Membrane & Vanadium ion permeability $\left(\times 10^{-12} \mathrm{~m}^{2} / \mathrm{s}\right)$ & Areal resistance $\left(\Omega \cdot \mathrm{cm}^{2}\right)$ \\
\hline PVDF30-B & $6.43 \pm 0.98$ & \multirow{2}{*}{$0.51 \pm 0.21$} \\
\hline PVDF30-T & $8.40 \pm 0.31$ & \\
\hline PVDF40-B & $1.46 \pm 0.44$ & \multirow{2}{*}{$0.93 \pm 0.12$} \\
\hline PVDF40-T & $3.82 \pm 0.87$ & \\
\hline PVDF50-B & $0.25 \pm 0.03$ & \multirow{2}{*}{$1.37 \pm 0.09$} \\
\hline PVDF50-T & $0.47 \pm 0.04$ & \\
\hline PVDF60-B & $0.32 \pm 0.11$ & \multirow{2}{*}{$12.17 \pm 0.21$} \\
\hline PVDF60-T & $0.44 \pm 0.25$ & \\
\hline PVDF70-B & $0.35 \pm 0.11$ & \multirow{2}{*}{$25.59 \pm 1.65$} \\
\hline PVDF70-T & $0.49 \pm 0.32$ & \\
\hline Nafion 115 & $0.66 \pm 0.09$ & $0.20 \pm 0.02$ \\
\hline $\mathrm{M}^{\mathrm{a}, \mathrm{R} 1}$ & 0.81 & 0.30 \\
\hline $\mathrm{M}-23-65^{\mathrm{b}, \mathrm{R} 2}$ & 1.70 & N/A \\
\hline $\mathrm{M}-23-100^{\mathrm{b}, \mathrm{R} 2}$ & 1.45 & N/A \\
\hline $\mathrm{M}-23-125^{\mathrm{b}, \mathrm{R} 2}$ & 1.32 & N/A \\
\hline Polycarbonate ${ }^{\mathrm{c}, \mathrm{R3}}$ & 2.51 & 0.24 \\
\hline
\end{tabular}

${ }^{a}$ prepared through NIPS process with PVDF/DMAc dope solution (16 wt.\% of PVDF), ${ }^{\text {b prepared }}$ through NIPS process with PVDF/DMAc dope solution (23 wt.\% of PVDF), but the thickness was varied from 65 to $125 \mu \mathrm{m}$, "track etched porous membrane having $50 \mathrm{~nm}$ cylindrical pores. 
Chemical stability test. The chemical stability of the membranes was performed under the strong oxidizing $\mathrm{VO}_{2}{ }^{+}\left(20 \mathrm{~mL}\right.$ of $0.1 \mathrm{M} \mathrm{VO}_{2}{ }^{+}$in $\left.5 \mathrm{M} \mathrm{H}_{2} \mathrm{SO}_{4}\right)$. If the membrane is chemically vulnerable, $\mathrm{VO}_{2}{ }^{+}$will be reduced to $\mathrm{VO}^{2+}$ over time, and the solution color will be changed from yellowish to greenish. To quantify the concentration changes of $\mathrm{VO}^{2+}$ from $\mathrm{VO}_{2}^{+}$, an UV-Vis spectroscopy (Agilent Technologies, Cary 8454) was conducted, and monitored the absorption of $\mathrm{VO}^{2+}$ at 760 $\mathrm{nm}$.

Table S3. Color change of $\mathrm{VO}_{2}{ }^{+}$solution containing PVDF membranes and Nafion at $40{ }^{\circ} \mathrm{C}$.

\begin{tabular}{|c|c|c|c|c|c|c|}
\hline Time (h) & PVDF30 & PVDF40 & PVDF50 & PVDF60 & Nafion & Blank \\
\hline 0 & & & & & & \\
\hline 291 & & & & & & \\
\hline 363 & & & & & & \\
\hline 456 & & & & & & \\
\hline 528 & & & & & & \\
\hline & & & & & & \\
\hline
\end{tabular}




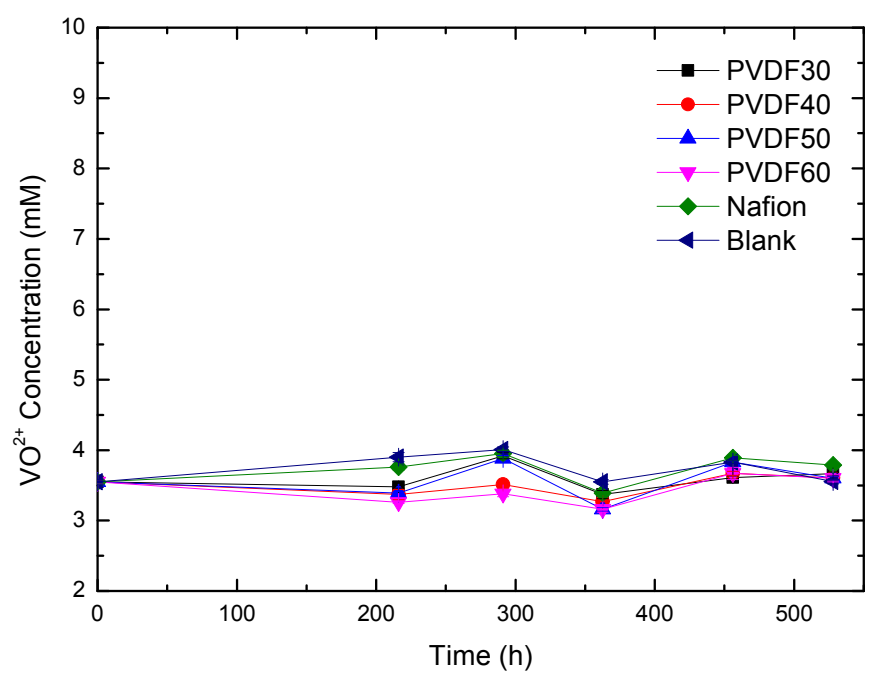

Figure S3. The transient $\mathrm{VO}^{2+}$ concentration in $20 \mathrm{~mL}$ of $0.1 \mathrm{M} \mathrm{VO}_{2}{ }^{+}$solution in $5 \mathrm{M} \mathrm{H}_{2} \mathrm{SO}_{4}$.

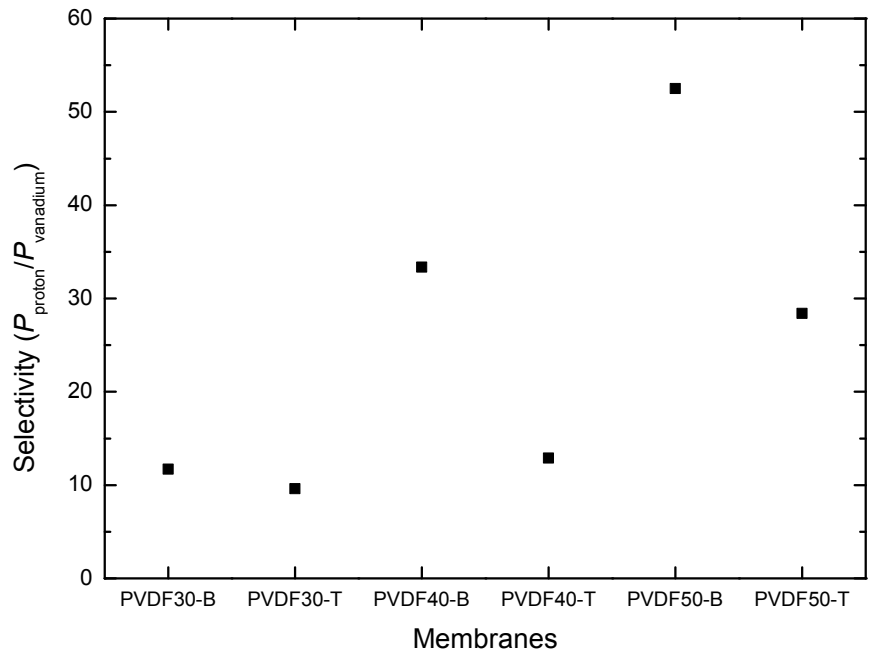

Figure S4. Proton selectivity to $\mathrm{VO}^{2+}$ for PVDF30, 40, 50 depending on the membrane direction toward the concentrated solution. Selectivity was evaluated by the ratio of proton permeability to vanadium permeability. 

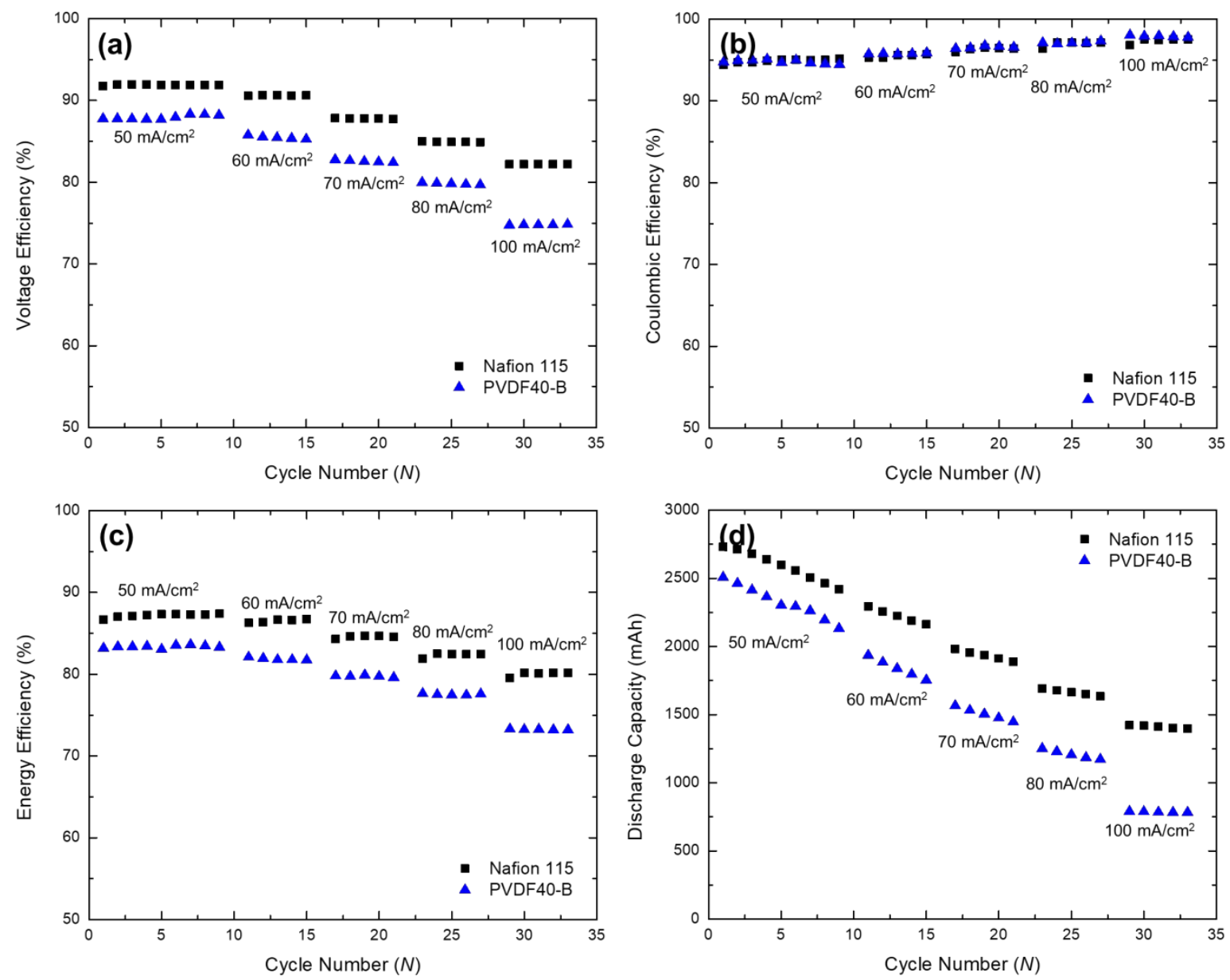

Figure S5. (a) Voltage efficiency, (b) Coulombic efficiency, (c) energy efficiency, and (d) discharge capacity of PVDF40-B, and Nafion 115 at various current densities, $50-100 \mathrm{~mA} / \mathrm{cm}^{2}$. 

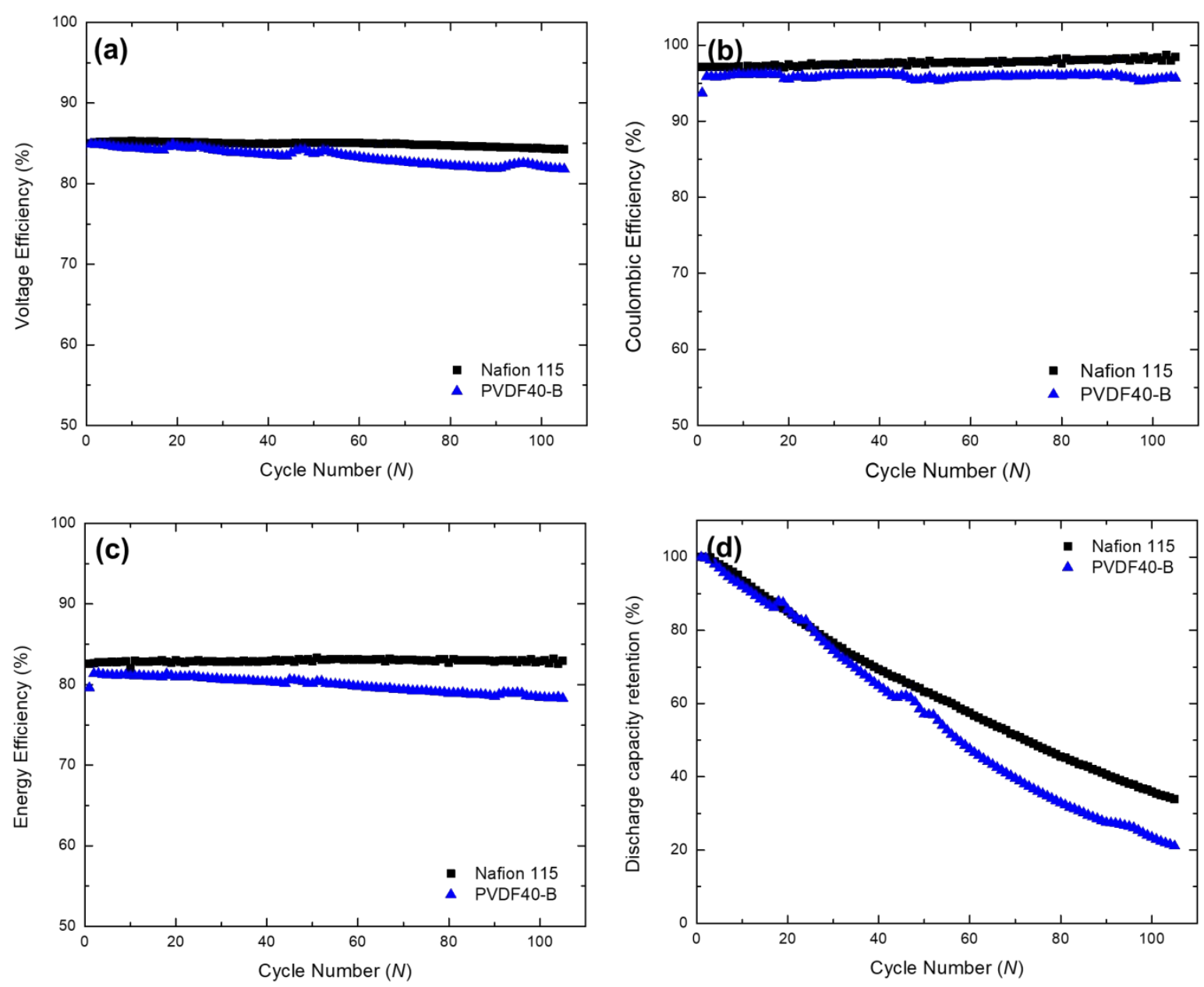

Figure S6. Long-term cycling efficiencies of PVDF40-B, and Nafion 115; (a) voltage efficiency, (b) Coulombic efficiency, (c) energy efficiency, and (d) discharge capacity retention capability. Current density was kept at $80 \mathrm{~mA} / \mathrm{cm}^{2}$. 
Table S4. VRFB cell performance at $80 \mathrm{~mA} / \mathrm{cm}^{2}$ with non-ion conducting porous membranes.

\begin{tabular}{ccccccc}
\hline Material & $\begin{array}{c}\text { Method for } \\
\text { pores }\end{array}$ & CE (\%) & VE (\%) & EE $(\%)$ & $\begin{array}{c}\text { Current density } \\
\left(\mathrm{mA} / \mathrm{cm}^{2}\right)\end{array}$ & Ref. \\
\hline PVDF & NIPS & 97.1 & 80.0 & 77.6 & 80 & This work \\
Nafion 115 & - & 96.4 & 85.0 & 81.9 & 80 & This work \\
PVDF & NIPS & 82.1 & 90.2 & 74.1 & 80 & r1 \\
PVDF & NIPS & 94.7 & 82.6 & 78.4 & 80 & r2 \\
PC & Track-etched & 75.0 & 62.0 & 47.0 & 80 & r3 \\
PAN & NIPS & 91.0 & 82.0 & 75.0 & 80 & r4 \\
\hline
\end{tabular}

\section{REFERENCES}

(R1) Cao, J.; Yuan, Z.; Li, X.; Xu, W.; Zhang, H. Hydrophilic Poly(Vinylidene Fluoride) Porous Membrane with Well Connected Ion Transport Networks for Vanadium Flow Battery. J. Power Sources 2015, 298, 228-235. https://doi.org/10.1016/j.jpowsour.2015.08.067.

(R2) Wei, W.; Zhang, H.; Li, X.; Zhang, H.; Li, Y.; Vankelecom, I. Hydrophobic Asymmetric Ultrafiltration PVDF Membranes: An Alternative Separator for VFB with Excellent Stability. Phys. Chem. Chem. Phys. 2013, 15 (6), 1766-1771. https://doi.org/10.1039/c2cp43761a.

(R3) Kim, J.; So, S.; Kim, H.-T.; Choi, S. Highly Ordered Ultrathin Perfluorinated Sulfonic Acid Ionomer Membranes for Vanadium Redox Flow Battery. ACS Energy Lett. 6 (1), 184-192. https://doi.org/10.1021/acsenergylett.0c02089.

(R4) Zhang, H.; Zhang, H.; Li, X.; Mai, Z.; Zhang, J. Nanofiltration (NF) Membranes: The next Generation Separators for All Vanadium Redox Flow Batteries (VRBs)? Energy Environ. Sci. 2011, 4 (5), 1676. https://doi.org/10.1039/clee01117k. 